\title{
In vivo measurement of tumor estradiol and Vascular Endothelial Growth Factor in breast cancer patients Stina Garvin and Charlotta Dabrosin*
}

\author{
Address: Linköping University, Division of Oncology, Faculty of Health Sciences, University Hospital, SE-581 85 Linköping, Sweden \\ Email: Stina Garvin - stina.garvin@lio.se; Charlotta Dabrosin* - chada@ibk.liu.se \\ * Corresponding author
}

Published: 18 March 2008

BMC Cancer 2008, 8:73 doi:10.1186/147|-2407-8-73

This article is available from: http://www.biomedcentral.com/I47/-2407/8/73

(c) 2008 Garvin and Dabrosin; licensee BioMed Central Ltd.

This is an Open Access article distributed under the terms of the Creative Commons Attribution License (http://creativecommons.org/licenses/by/2.0), which permits unrestricted use, distribution, and reproduction in any medium, provided the original work is properly cited.
Received: 17 August 2007

Accepted: 18 March 2008

\begin{abstract}
Background: Angiogenesis, crucial for tumor progression, is a process regulated in the tissue micro-environment. Vascular endothelial growth factor (VEGF) is a potent stimulatory factor of angiogenesis and a negative prognostic indicator of breast cancer. VEGF is biologically active in the extracellular space and hitherto, there has been a lack of techniques enabling sampling of angiogenic molecules such as VEGF in situ. The majority of breast cancers are estrogen-dependent, and estrogen has been shown to regulate VEGF in normal breast tissue and experimental breast cancer. We investigated if microdialysis may be applicable in human breast cancer for sampling of extracellular VEGF in situ and to explore if there is an association with local estradiol and VEGF levels in normal and cancerous breast tissue.
\end{abstract}

Methods: Microdialysis was used to sample VEGF and estradiol in tumors and adjacent normal breast tissue in postmenopausal breast cancer patients. VEGF and estradiol were also measured in plasma, and immunohistochemical staining for VEGF was performed on tumor sections.

Results: We show that in vivo levels of extracellular VEGF were significantly higher in breast cancer tumors than in normal adjacent breast tissue. There was a significant positive correlation between estradiol and extracellular VEGF in normal breast tissue. However, no correlation was detected between estradiol and VEGF in tumors or between tumor VEGF and plasma VEGF.

Conclusion: We conclude that VEGF and estradiol correlates significantly in normal breast tissue. Microdialysis may be used to provide novel insight in breast tumor biology and the regulation of molecules in the extracellular space of human breast tumors in vivo.

\section{Background}

Vascular endothelial growth factor (VEGF) is a potent mediator of tumor angiogenesis, including neovascularization in human breast cancer [1]. In breast cancer patients, high tumor VEGF levels, as assessed by immunohistochemistry or quantitative immunoassay, appear to correlate with poor prognosis and decreased overall survival for both node-positive and node-negative breast can- cer patients [2-4]. VEGF exists in several isoforms; the shorter isoforms are diffusible proteins whereas the longer isoforms are sequestered in the extracellular matrix [5]. Proteolytic cleavage may convert the longer VEGF isoforms into soluble, bioactive forms in the extracellular space where they become available to endothelial cells [5]. Sex steroids have been shown to increase VEGF expression in normal breast tissue as well as in experimen- 
tal breast cancer, both in vitro and in vivo [6-10], and an estrogen responsive element (ERE) has been identified in the promoter region of the gene for VEGF [11].

Estrogen exposure is considered a major risk factor for development of breast cancer and the majority of breast cancers maintain their hormonal dependency $[12,13]$. After menopause the circulating levels of estradiol are greatly reduced, yet postmenopausal women have been shown to maintain breast tissue estradiol levels higher than corresponding plasma levels [14]. Moreover, levels of estradiol in breast tumor tissue have been shown to be higher than those in normal breast tissue distant from the tumor [14,15], and the major enzymes involved in estrogen synthesis - aromatase, sulphatase, and $17 \beta$-hydroxysteroid dehydrogenase - have been demonstrated in human breast cancer [16-18]. As VEGF may be released and estradiol metabolized locally in the breast, an in situ technique for surveying these molecules may provide novel insight into their regulation in tumor tissue in vivo. We have previously used microdialysis for in vivo measurements of estradiol, VEGF, and other molecules in normal human breast and experimental breast cancer [6$8,19-21]$. In this study we have performed microdialysis in breast cancer patients in order to sample estradiol and VEGF locally in breast cancer tumors and in adjacent normal breast tissue. We found that extracellular levels of VEGF were significantly higher in tumors than in normal breast tissue in vivo. In addition, we show a positive correlation between normal breast tissue and plasma levels of estradiol and extracellular VEGF in normal breast tissue in vivo.

\section{Methods \\ Subjects}

Ten postmenopausal women, ages 51-86, participated in the study. Postmenopausal status was defined by having no spontaneous menstrual bleeding for at least a year in women older than 50 . None of the women had ongoing hormonal treatment. The study was approved by the local ethical review board in Linköping, Sweden, (reference number 03-081) and performed in compliance with the
Helsinki Declaration, and all women gave informed consent. Patient characteristics are provided in Table 1.

\section{Microdialysis}

Microdialysis was performed on the day before or the same day as surgery using microdialysis catheters with a molecular weight cut-off of 100,000 Da (CMA Microdialysis AB, Stockholm, Sweden). Each catheter consists of a tubular dialysis membrane (length $10 \mathrm{~mm}$; diameter 0.52 $\mathrm{mm}$ ) attached to a double-lumen tube (length $100 \mathrm{~mm}$; diameter $0.8 \mathrm{~mm}$ ). Prior to microdialysis, mepivacaine $(0.3 \mathrm{~mL} ; 5 \mathrm{mg} / \mathrm{mL})$ was administered intracutaneously as a local anesthetic. Two microdialysis catheters were thereafter inserted in the affected breast, one intratumorally and one in adjacent, macroscopically normal breast tissue. The insertion was guided by catheters for intravenous use (Venflon, $1.4 \mathrm{~mm}$; BOC Ohmeda AB, Helsingborg, Sweden). The microdialysis catheters were connected to a microinfusion pump (CMA 107, CMA Microdialysis AB) and perfused with $\mathrm{NaCl}(154 \mathrm{mM})$ and dextran-70 (40 g/ $\mathrm{L})$ at a rate of $0.5 \mu \mathrm{L} / \mathrm{min}$. After an equilibration period of 30 minutes, the outgoing dialysate was collected and stored together with plasma samples at $-70^{\circ} \mathrm{C}$ for subsequent analysis. No complications occurred during or after the microdialysis experiments.

Microdialysis allows sampling of the extracellular fluid by passive diffusion of substances over a semi-permeable membrane. The recovery i.e. the amount of substances that diffuse into the perfusion fluid depends on the tissue and membrane properties, the flow rate, and the size of the compound of interest [22]. Diffusion of low molecular substances over the dialysis membrane is almost complete at low flow rates using a $30 \mathrm{~mm}$ long dialysis membrane [23]. However, for larger molecules the recovery over the membrane decreases and the measured levels in the microdialysis sample may not be absolute concentrations in the tissue. The recovery of certain substance may be measured in vitro, however, this in vitro recovery can only be an estimate of the in vivo recovery since other factors such as tissue pressure and temperature will affect the diffusion of substances. For the in vivo recovery several

Table I: Patient and tumor characteristics

\begin{tabular}{|c|c|c|c|c|c|c|c|c|c|c|}
\hline & I & 2 & 3 & 4 & 5 & 6 & 7 & 8 & 9 & 10 \\
\hline Age & 67 & 59 & 51 & 86 & 83 & 66 & 56 & 68 & 65 & 57 \\
\hline Histology & lobular & ductal & ductal & ductal & ductal & ductal & ductal & lobular & lobular & ductal \\
\hline Size $(\mathrm{mm})$ & 18 & 9 & 20 & 60 & 20 & 20 & 17 & 15 & 50 & 21 \\
\hline Disease stage $(T)$ & 1 & 1 & 1 & 3 & 2 & 2 & 1 & 1 & 2 & 2 \\
\hline Histological grade (NHG) & 2 & 2 & 3 & 3 & 2 & 2 & 2 & 2 & 2 & 3 \\
\hline ER $(\%)$ & 50 & 80 & 100 & 80 & 100 & 80 & 100 & 75 & 75 & 75 \\
\hline PR (\%) & 100 & 10 & 0 & 80 & 100 & 0 & 0 & 0 & 50 & 0 \\
\hline
\end{tabular}

Abbreviations: $\mathrm{ER}=$ estrogen receptor, $\mathrm{PR}=$ progesterone receptor 
calibration techniques such as the no net flux technique, the stop flow techniques, and the endogenous reference technique have been developed for low molecular substances unbound in the extracellular space. However, when measuring high molecular weight proteins these techniques may not be applicable because the proteins of interest may be bound to binding proteins in vivo, recombinant proteins may not have the same properties as the natural proteins in vivo, and plasma and tissue levels of the protein are not equal. We have previously found that in the case of VEGF tissue homogenate, unlike plasma VEGF, correlate significantly with VEGF sampled by microdialysis. We have previously reported an in vitro recovery of VEGF of $8 \%$ and an in vitro recovery of estradiol from plasma of $30 \%$ and these data were confirmed in the present study $[7,20]$. In the present study all microdialysis values are given as original raw data without any re-calculations.

\section{Quantification of estradiol and VEGF}

Measurements of estradiol in plasma and microdialysis dialysate were conducted using a commercial quantitative immunoassay kit (estradiol ELISA, DRG Instruments $\mathrm{GmbH}$, Marburg, Germany) according to the manufacturer's instructions. VEGF was quantified using a commercial quantitative immunoassay kit for human VEGF (Fluorokine MAP human VEGF kit, R\&D systems, Abingdon, UK) and analyzed by the Luminex 100 IS System (Luminex B.V., Oosterhout, Netherlands). According to the manufacturer, the VEGF kit measures the VEGF $_{165}$ and VEGF $_{121}$ isoforms, and the mean minimum detectable dose is $0.81 \mathrm{pg} / \mathrm{mL}$. The intra-assay precision for this kit is $3.7-7 \%$ which was confirmed during the experiment. All microdialysis values are stated as raw data.

\section{Immunohistochemistry of tumor sections}

Formalin-fixed, paraffin-embedded tumors were sectioned, deparaffinized and subjected to anti-human VEGF immunohistochemistry (monoclonal mouse anti-human VEGF, specific for the VEGF $_{165}$ and VEGF $_{121}$ isoforms, dilution 1:20, R\&D systems, with Envision detection, DAKO). Sections were counterstained with Mayer's hematoxylin. Negative controls did not show staining. In a blinded manner, entire sections were first scanned to determine the range of intensity of the staining. VEGF staining was thereafter scored as weakly or strongly positive. Estrogen receptor (ER) and progesterone receptor (PR) were determined by immunohistochemical staining using the Ventana Benchmark system (Ventana Medical Systems Inc., Arizona, USA) and defined as the fraction of positive nuclei. Monoclonal rabbit anti-human estrogen receptor alpha antibody (prediluted 1:50, Lab Vision Ltd, Suffolk, UK) and monoclonal mouse anti-human progesterone receptor antibody (prediluted 1:50, Novocastra Laboratories Ltd, Newcastle, UK) were used for staining.

\section{Clinicopathological data}

Tumor histology, size, stage (T of the TNM classification), and Nottingham histological grade (NHG) according to Elston Ellis scoring system were determined at the Department of Pathology and Cytology, University Hospital of Linköping.

\section{Statistical analysis}

Shapiro-Wilks' W test for normality was used. Wilcoxons' signed rank test for paired observations was used and normally distributed data was calculated using Pearson's correlation coefficient. Results are given in median [25-75 precentile].

\section{Results}

\section{Characteristics of patients and tumors}

Ten patients were included in the study, 7 with invasive ductal carcinoma and 3 with invasive lobular carcinoma. All of the tumors were ER-positive; 5 of 10 were PR-positive. Tumors were stage pT1-3 (TNM) and NHG 2-3. All tumors showed positive scoring for mitosis (not shown). Clinicopathological data is provided in Table 1.

\section{Extracellular VEGF significantly higher in breast cancer tumors than in adjacent normal breast tissue}

Using microdialysis, levels of estradiol and extracellular VEGF were measured in tumors and in adjacent normal breast tissue in vivo. Levels of intratumoral VEGF were significantly higher than in normal breast tissue, $6.0 \mathrm{pg} / \mathrm{mL}$ [4.1-6.5] in tumors compared to $2.4 \mathrm{pg} / \mathrm{mL}[2,3]$ in breast tissue, $p=0.005$, Wilcoxon signed rank test, Figure 1 ). Plasma levels of VEGF were $3.67 \mathrm{pg} / \mathrm{mL}$ [0.8-5.7], range $0.62-7.59 \mathrm{pg} / \mathrm{mL}$. In 7 of the 10 patients tumor estradiol levels were higher than in normal breast tissue, however, when comparing the whole group no significant differences were found between the levels of tumor estradiol, $106 \mathrm{pM}$ [72-136], and normal breast tissue estradiol, 87 pM [48-150], Figure 2. Plasma levels of estradiol were 60 pM [40-84], range 24-198 pM.

\section{Significant correlation between normal breast estradiol and breast VEGF in vivo}

A significant positive correlation was found between plasma levels and normal breast tissue levels of estradiol, Figure 3. No correlations were found between tumor estradiol and tumor VEGF or between plasma VEGF and tumor VEGF. ER, PR, and tumor size did not correlate with VEGF or estradiol measured in microdialysis dialysate or plasma.

\section{Immunohistochemistry of VEGF}

All tumor sections showed positive intracellular staining for VEGF. Inter-individual differences in intratumoral VEGF quantified in microdialysis dialysate were not reflected in the intensity of staining for VEGF. 


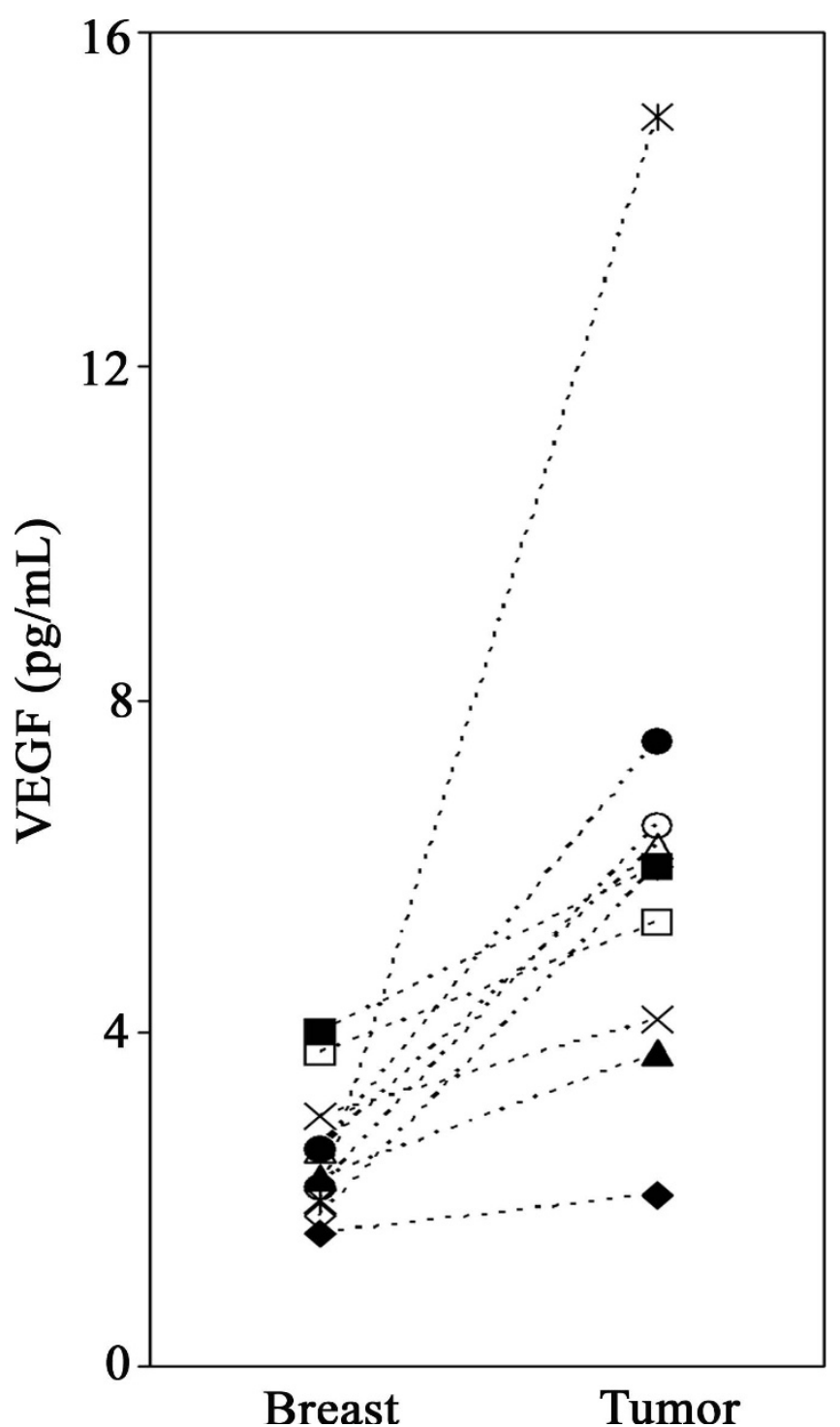

Figure I

Extracellular VEGF in normal breast and tumors of postmenopausal breast cancer patients. VEGF sampled in vivo by microdialysis was significantly higher in tumors than in adjacent normal breast tissue $(p=0.005)$. Each symbol represents an individual patient.

\section{Discussion}

To our knowledge this is the first report of intratumoral sampling of extracellular estradiol and VEGF in vivo in breast cancer patients. We show that tumor levels of extracellular VEGF were significantly higher than those in adjacent normal breast tissue in vivo. In addition, we show a positive correlation between normal breast tissue levels of estradiol and extracellular VEGF in normal breast tissue in vivo, in agreement with previous findings in a larger cohort of healthy women [6].

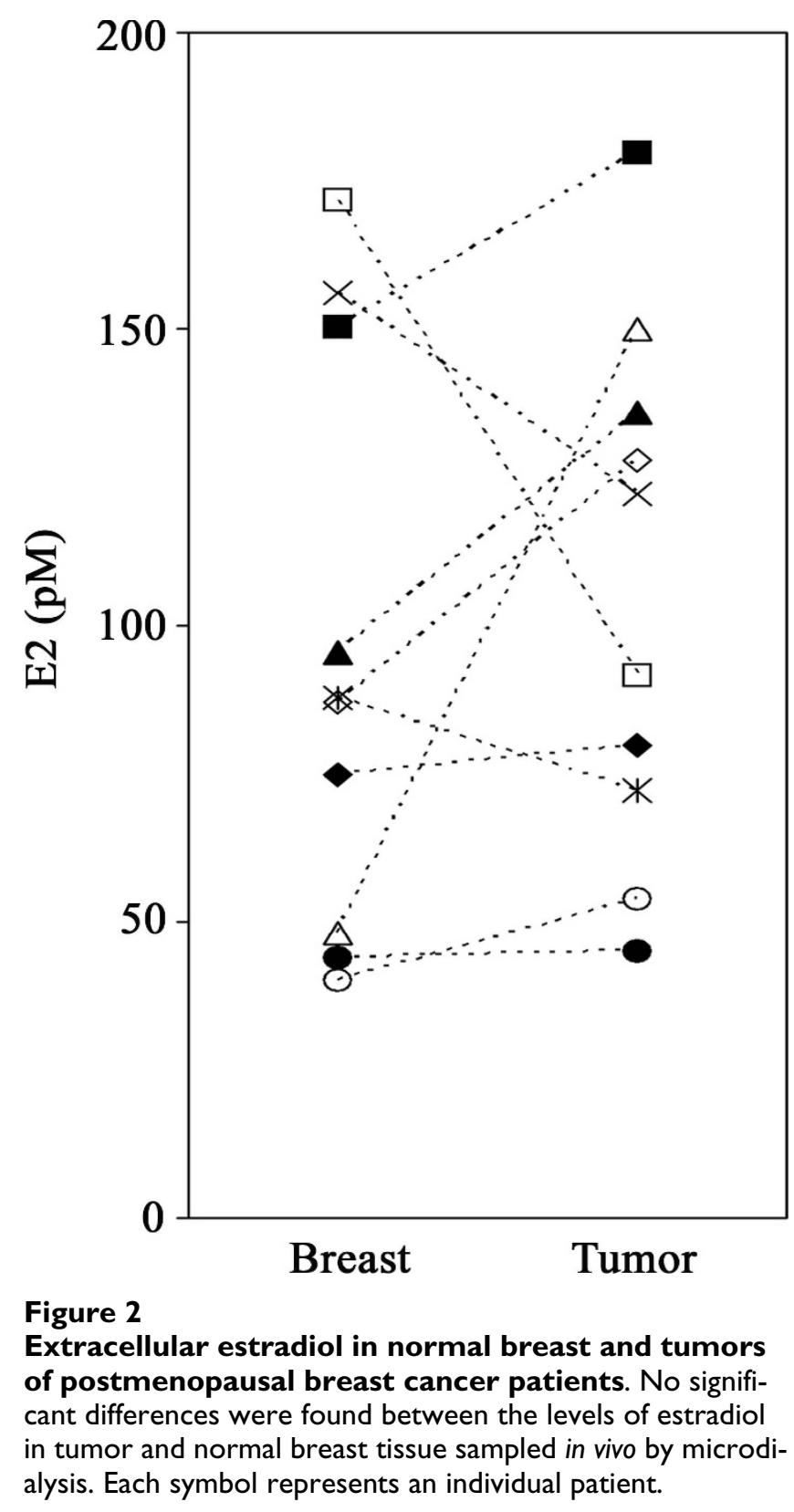

Higher extracellular VEGF in tumor tissue compared to normal tissue is in line with previous ex vivo findings demonstrating an up-regulation of VEGF in breast cancer compared to adjacent non-neoplastic tissue $[24,25]$. The interindividual differences in extracellular VEGF measured in vivo were not apparent in the ex vivo immunohistochemical staining. This may be explained by the fact that the shorter soluble isoforms of VEGF, which are sampled using microdialysis, are not likely to be detected in the extracellular space by immunostaining of tissue sections [26]. The freely diffusible $\mathrm{VEGF}_{121}$ isoform has been shown to be more angiogenic and tumorigenic than other splice variants of VEGF and is considered the predomi- 


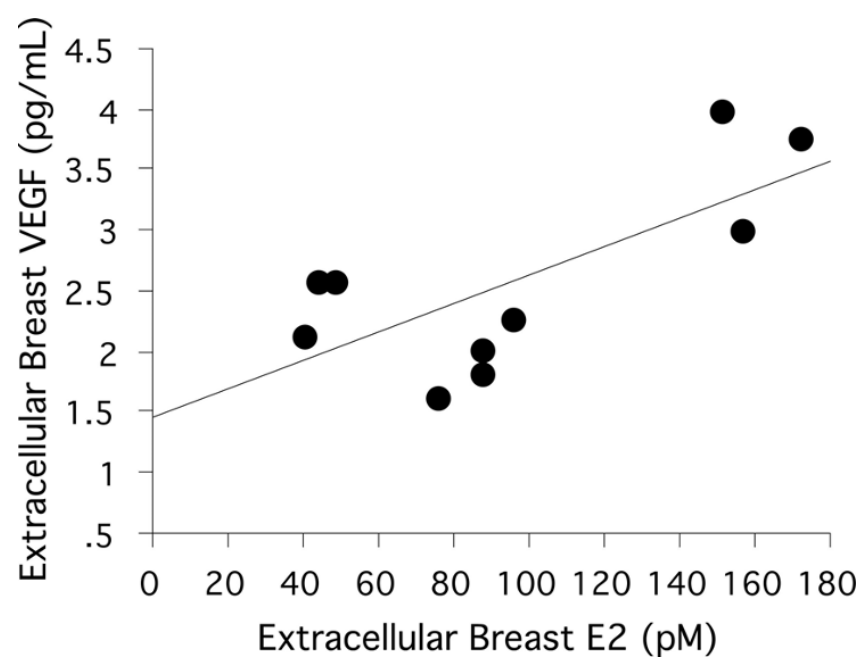

Figure 3

Correlation of local extracellular breast VEGF and estradiol in normal breast tissue of breast cancer patients. There was a significant correlation between extracellular local breast estradiol (E2) and VEGF in normal breast tissue of breast cancer patients, $(r=0.708, p=0.022)$.

nant isoform in human breast cancer [26,27]. Moreover, our previous studies suggest that quantitative differences in secreted VEGF are not necessarily detectable in semiquantitative immunohistochemical staining $[6,8,9]$. Hence, sampling VEGF in the extracellular space, where it exerts its effects, may add significant insight into its regulation in tumor tissue.

Previous ex vivo studies have shown that estradiol levels may be higher in tumor tissue than in normal breast tissue distant from the tumor $[14,15]$. The demonstration of enzymes involved in estrogen synthesis in human breast cancer has led to the intracrine concept that local estrogens produced in a breast tumor and surrounding tissue contributes to tumor development and progression [1618]. In this study, estradiol levels measured in tumors in vivo were indeed higher than in normal breast tissue in 7 of the 10 patients with up to three times higher levels of estradiol in tumors, supporting possible intratumoral estrogen production in some breast cancers.

While a significant correlation was found between normal tissue estradiol and VEGF, there was no correlation between levels of tumor estradiol and VEGF. Although all tumors were ER positive, levels of expression varied between patients and half of the tumors showed no PR expression. Loss of PR expression has previously been attributed to a non-functional ER [28], which may be one reason for the absence of correlation between tumor estradiol and tumor VEGF. Therefore our results do not rule out a possible regulation of VEGF by estradiol in breast cancer tumors. Hypoxia is a potent regulator of VEGF and tumor angiogenesis via hypoxia inducible factor-I $[29,30]$, and we have previously demonstrated a positive correlation between tumor size and VEGF in experimental breast cancer models $[8,31]$. Xenografts derived from cell lines are however more homogeneous than human tumors due to the use of only one clone of cancer cell [32]. In this study both lobular and ductal carcinomas were included, and tumor size varied between 9-60 $\mathrm{mm}$. The heterogeneity and the small sample size may explain, at least in part, the lack of correlation between tumor size and tumor VEGF.

Plasma and tumor levels of VEGF did not correlate in this study in line with our previous findings demonstrating that less than half of the VEGF in circulating plasma originates from the tumor [7]. This suggests that determination of VEGF in plasma is a poor indicator of tumor VEGF.

The fact that estradiol may be synthesized and VEGF mobilized locally in the breast and in breast cancer emphasizes the need to sample these molecules in the extracellular space. This study is the first of its kind to measure VEGF and estradiol in human breast cancer tumors in situ by microdialysis. Sampling regulators of the tumor microenvironment directly in situ is highly desirable taking into consideration that gene expression levels and intracellular protein levels are not always indicative of biologically active extracellular protein levels [8]. It may be argued that the differences seen between tumor and normal tissue merely reflect differences in recovery between the tissues. However, tumor tissue have increased blood flow compared to normal breast tissue which in theory would decrease the recovery and result in decreased levels of the compounds in the tumor. In our present study tumor tissue exhibited higher levels of VEGF. Hence, these results cannot be explained by possible differences of the in vivo recovery only. Moreover, correlations are measured in the same sample from the same tissue and these results are unaffected by different tissue recoveries. While studies today are generally limited to in vitro cell culture methods, animal models, and ex vivo surgical specimens, microdialysis is emerging as unique tool for in vivo sampling in accessible tumors such as those of the breast [33].

\section{Conclusion}

Our findings show that the in vivo levels of extracellular VEGF were significantly higher in breast cancer tumors than in normal breast tissue. In addition, our results support the role of estradiol as a potent regulator of VEGF in normal breast tissue in vivo. Further studies are warranted to investigate the role of VEGF in mediating sex steroid effects on breast cancer development and progression. 
Novel in situ data provided by microdialysis may increase our understanding of breast cancer tumor biology.

\section{Competing interests}

The author(s) declare that they have no competing interests.

\section{Authors' contributions}

$\mathrm{CD}$ conceived and designed the study. Microdialysis and data collection were performed by CD and SG. SG performed data analysis and SG and CD co-wrote the manuscript. Both authors read and approved the final manuscript.

\section{Acknowledgements}

This study was supported by grants from the Swedish Cancer Society, the Swedish Research Council, and Linköping University Hospital. The authors would like to express their gratitude to the staff of the Division of Surgery, Linköping University Hospital, for their assistance in this study.

\section{References}

I. Folkman J: Angiogenesis in cancer, vascular, rheumatoid and other disease. Nat Med 1995, I:27-31.

2. Linderholm B, Grankvist K, Wilking N, Johansson M, Tavelin B, Henriksson R: Correlation of vascular endothelial growth factor content with recurrences, survival, and first relapse site in primary node-positive breast carcinoma after adjuvant treatment. I Clin Oncol 2000, 18:1423-1431.

3. Linderholm B, Tavelin B, Grankvist K, Henriksson R: Vascular endothelial growth factor is of high prognostic value in nodenegative breast carcinoma. J Clin Oncol 1998, 16:3 I2I-3 I 28.

4. Toi M, Inada $\mathrm{K}$, Suzuki $\mathrm{H}$, Tominaga $\mathrm{T}$ : Tumor angiogenesis in breast cancer: its importance as a prognostic indicator and the association with vascular endothelial growth factor expression. Breast Cancer Res Treat 1995, 36:193-204.

5. Ferrara N, Davis-Smyth T: The biology of vascular endothelial growth factor. Endocr Rev 1997, 18:4-25.

6. Dabrosin C: Positive correlation between estradiol and vascular endothelial growth factor but not fibroblast growth factor-2 in normal human breast tissue in vivo. Clin Cancer Res 2005, I I:8036-804I.

7. Dabrosin C, Margetts PJ, Gauldie J: Estradiol increases extracellular levels of vascular endothelial growth factor in vivo in murine mammary cancer. Int J Cancer 2003, 107:535-540.

8. Garvin S, Dabrosin C: Tamoxifen inhibits secretion of vascular endothelial growth factor in breast cancer in vivo. Cancer Res 2003, 63:8742-8748.

9. Garvin S, Nilsson UW, Huss FR, Kratz G, Dabrosin C: Estradiol increases VEGF in human breast studied by whole-tissue culture. Cell Tissue Res 2006, 325:245-25I.

10. Hyder SM, Murthy L, Stancel GM: Progestin regulation of vascular endothelial growth factor in human breast cancer cells. Cancer Res 1998, 58:392-395.

II. Hyder SM, Nawaz Z, Chiappetta C, Stancel GM: Identification of functional estrogen response elements in the gene coding for the potent angiogenic factor vascular endothelial growth factor. Cancer Res 2000, 60:3183-3190.

12. Hulka BS, Stark AT: Breast cancer: cause and prevention. Lancet 1995, 346:883-887.

13. Rossouw JE, Anderson GL, Prentice RL, LaCroix AZ, Kooperberg C, Stefanick ML, Jackson RD, Beresford SA, Howard BV, Johnson KC, et al.: Risks and benefits of estrogen plus progestin in healthy postmenopausal women: principal results From the Women's Health Initiative randomized controlled trial. Jama 2002, 288:32I-333.

14. Vermeulen A, Deslypere JP, Paridaens R, Leclercq G, Roy F, Heuson IC: Aromatase, 17 beta-hydroxysteroid dehydrogenase and intratissular sex hormone concentrations in cancerous and normal glandular breast tissue in postmenopausal women. Eur J Cancer Clin Oncol 1986, 22:5I5-525.
15. van Landeghem AA, Poortman J, Nabuurs M, Thijssen JH: Endogenous concentration and subcellular distribution of estrogens in normal and malignant human breast tissue. Cancer Res 1985, 45:2900-2906.

16. Geisler J: Breast cancer tissue estrogens and their manipulation with aromatase inhibitors and inactivators. J Steroid Biochem Mol Biol 2003, 86:245-253.

17. Gunnarsson C, Olsson BM, Stal O: Abnormal expression of I7beta-hydroxysteroid dehydrogenases in breast cancer predicts late recurrence. Cancer Res 200 I, 6 I:8448-845 I.

18. Pasqualini JR, Chetrite GS: Recent insight on the control of enzymes involved in estrogen formation and transformation in human breast cancer. J Steroid Biochem Mol Biol 2005, 93:221-236

19. Dabrosin C: Increase of free insulin-like growth factor-I in normal human breast in vivo late in the menstrual cycle. Breast Cancer Res Treat 2003, 80:193-198.

20. Dabrosin C: Increased extracellular local levels of estradiol in normal breast in vivo during the luteal phase of the menstrual cycle. J Endocrinol 2005, 187:103-108.

21. Dabrosin C: Variability of Vascular Endothelial Growth Factor in Normal Human Breast Tissue in Vivo during the Menstrual Cycle. J Clin Endocrinol Metab 2003, 88:2695-2698.

22. Ungerstedt U: Microdialysis - principles and applications for studies in animals and man. J Intern Med 1991, 230:365-373.

23. Rosdahl H, Hamrin K, Ungerstedt U, Henriksson J: Metabolite levels in human skeletal muscle and adipose tissue studied with microdialysis at low perfusion flow. Am J Physiol 1998, 274:E936-945.

24. Brown LF, Berse B, Jackman RW, Tognazzi K, Guidi AJ, Dvorak HF, Senger DR, Connolly JL, Schnitt SJ: Expression of vascular permeability factor (vascular endothelial growth factor) and its receptors in breast cancer. Hum Pathol 1995, 26:86-91.

25. Yoshiji H, Gomez DE, Shibuya M, Thorgeirsson UP: Expression of vascular endothelial growth factor, its receptor, and other angiogenic factors in human breast cancer. Cancer Res 1996, 56:2013-2016.

26. Zhang HT, Scott PA, Morbidelli L, Peak S, Moore J, Turley H, Harris $A L$, Ziche $M$, Bicknell $R$ : The I 2 I amino acid isoform of vascular endothelial growth factor is more strongly tumorigenic than other splice variants in vivo. Br J Cancer 2000, 83:63-68.

27. Relf M, Lejeune S, Scott PA, Fox S, Smith K, Leek R, Moghaddam A Whitehouse R, Bicknell R, Harris AL: Expression of the angiogenic factors vascular endothelial cell growth factor, acidic and basic fibroblast growth factor, tumor growth factor beta-I, platelet-derived endothelial cell growth factor, placenta growth factor, and pleiotrophin in human primary breast cancer and its relation to angiogenesis. Cancer Res 1997, 57:963-969.

28. Horwitz KB, Koseki Y, McGuire WL: Estrogen control of progesterone receptor in human breast cancer: role of estradiol and antiestrogen. Endocrinology 1978, 103:1742-175I.

29. Forsythe JA, liang BH, lyer NV, Agani F, Leung SW, Koos RD, Semenza GL: Activation of vascular endothelial growth factor gene transcription by hypoxia-inducible factor I. Mol Cell Biol 1996, 16:4604-4613.

30. Pugh CW, Ratcliffe PJ: Regulation of angiogenesis by hypoxia: role of the HIF system. Nat Med 2003, 9:677-684.

31. Dabrosin C, Chen J, Wang L, Thompson LU: Flaxseed inhibits metastasis and decreases extracellular vascular endothelial growth factor in human breast cancer xenografts. Cancer Lett 2002, 185:3I-37.

32. Lacroix M, Leclercq G: Relevance of breast cancer cell lines as models for breast tumours: an update. Breast Cancer Res Treat 2004, 83:249-289.

33. Dabrosin C: Microdialysis - an in vivo technique for studies of growth factors in breast cancer. Front Biosci 2005, I 0: | 329- | 335.

\section{Pre-publication history}

The pre-publication history for this paper can be accessed here:

http://www.biomedcentral.com/1471-2407/8/73/prepub 